\title{
Supporting Information for: Spectral Reshaping of Single Dye Molecules Coupled to Single Plasmonic Nanoparticles
}

\author{
Stephen A. Lee ${ }^{1}$ and Julie S. Biteen ${ }^{1, *}$
}

${ }^{1}$ Department of Chemistry, University of Michigan, Ann Arbor, Michigan 48109, United States 


\section{Supporting Methods:}

Finite Difference Time Domain (FDTD) Simulations

Electromagnetic simulations were performed using Lumerical FDTD Solutions software. The reshaped spectrum of the coupled dye/AuNP pair, $F(r, \omega)$, was calculated according to Ringler et al. ${ }^{1}$ This coupled spectrum was calculated for each dye at various Dye/AuNP separations, $r$, (main text Fig. 1b) based on $F_{0}(\omega)$, the intrinsic fluorescence emission spectrum of BDP-FL, BDP-R6G, Cy3, or Cy3.5 (Eqs. S1 - S3):

$$
F(r, \omega)=F_{0}(\omega) \times g_{e x}\left(r, \omega_{L}\right) \times g_{e m}(r, \omega)
$$

Here, $g_{e x}$ is the excitation enhancement function and $g_{e m}$ is the emission enhancement function. The excitation enhancement function is calculated at the laser frequency, $\omega_{L}$ :

$$
g_{e x}\left(r, \omega_{L}\right)=\frac{\sum_{i=x, y, z} E_{i}^{2}\left(r, \omega_{L}\right)}{\sum_{i=x, y, z} E_{0 i}^{2}\left(r, \omega_{L}\right)}
$$

where $E_{i}^{2}$ is the $x-, y$-, or $z$-component of the electric field intensity, and the emission enhancement function is calculated as:

$$
g_{e m}(r, \omega)=\frac{1}{\eta_{o}} \frac{\frac{P_{r}(r, \omega)}{P_{0 r}(r, \omega)}}{\int_{0}^{\infty} f(\omega) \frac{P_{r}(r, \omega)}{P_{0 r}(r, \omega)} d \omega+\int_{0}^{\infty} f(\omega) \frac{P_{t o t}(r, \omega)-P_{r}(r, \omega)}{P_{0 r}(r, \omega)} d \omega+\frac{1}{\eta_{o}}-1}
$$

where $P_{r}$ is the power radiated by a dipole source in the presence of the AuNP and $P_{0 r}$ is the power radiated by a dipole source in the absence of the AuNP, $P_{\text {tot }}$ is the Purcell enhancement of a dipole near the AuNP, $f(\omega)$ is the integral normalized intrinsic emission spectrum of the dye, and $\eta_{o}$ is the intrinsic quantum yield of the dye. Per Ringler et al. ${ }^{1}$, the total enhancement function is assumed to be linear, such that the enhancement at one frequency does not affect the enhancement at other frequencies.

In the FDTD calculations, $P_{\text {tot }}$ is measured as the total power transmitted through a box around the dipole source (green box in Fig. 1b) and $P_{r}$ is the power transmitted through a box around the source and the AuNP (purple box in Fig. 1b). ${ }^{2,3}$

The resulting excitation factor is presented as a function of $r$ in Fig. S5a. The emission enhancement functions, $g_{e m}$, are plotted as a function of distance and wavelength in Fig. S5b - e. The greatest changes in the emission reshaping occur for separation distances $<150 \mathrm{~nm}$. 
Supporting Figures
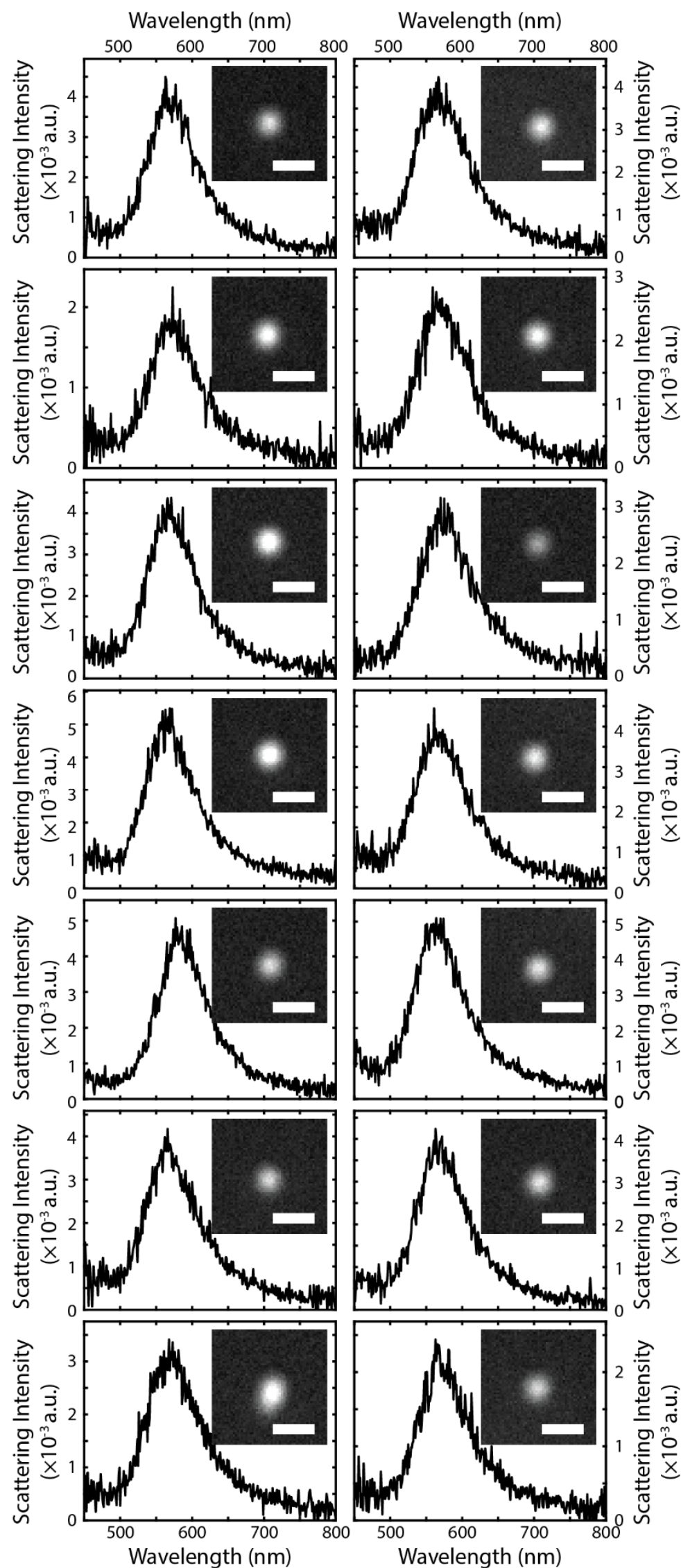

Figure S1. Single-particle dark-field spectra of the 14 nanoparticles studied. The normalized spectra were aggregated to produce the grey curve in main text Figure 1a. Insets: dark-field scattering images of each particle. Scale bars: $1 \mu \mathrm{m}$. 


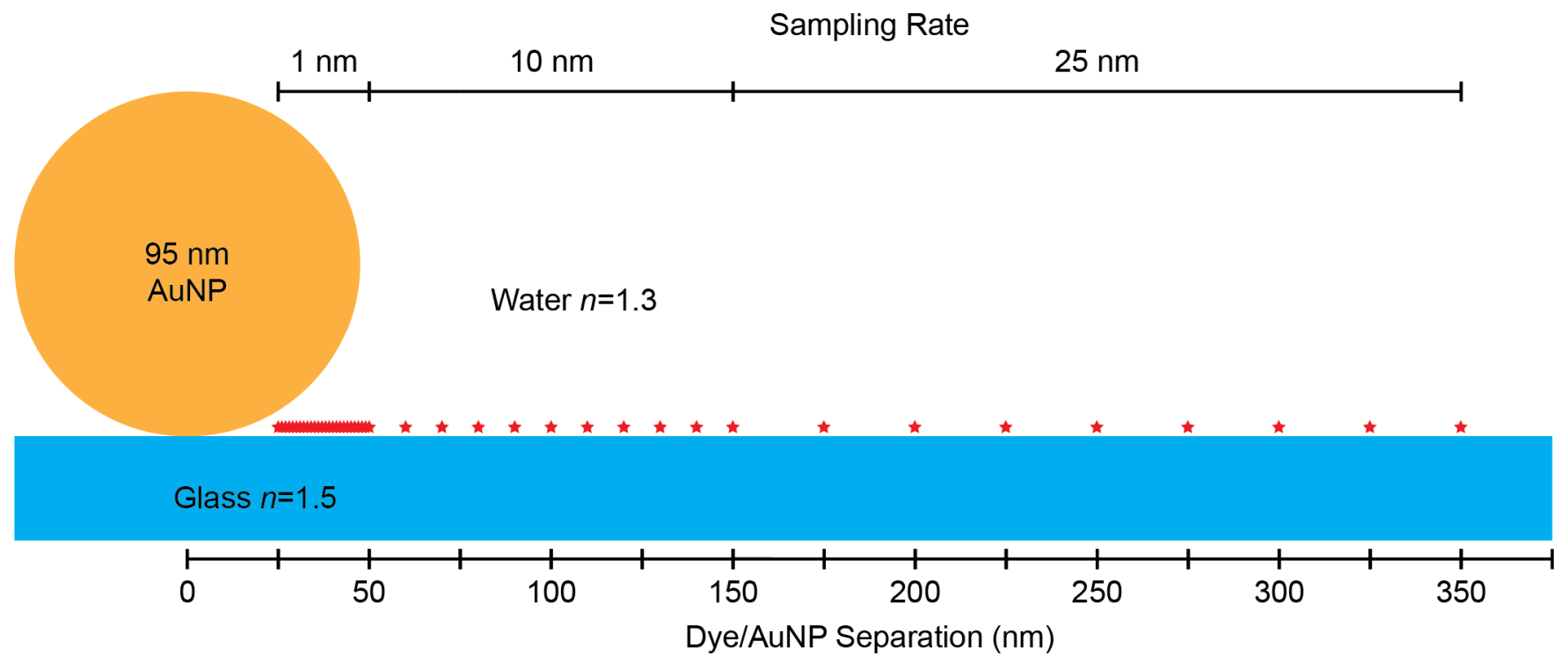

Figure S2. Side view of simulated dye positions relative to the positions of the AuNP and glass surface. Point electric dipoles (red stars) were simulated at regular positions, $r$, according to the sampling rate, next to a gold sphere with a diameter of $95 \mathrm{~nm}$. The read-out of these simulations is described in main text Fig. $1 b$. 

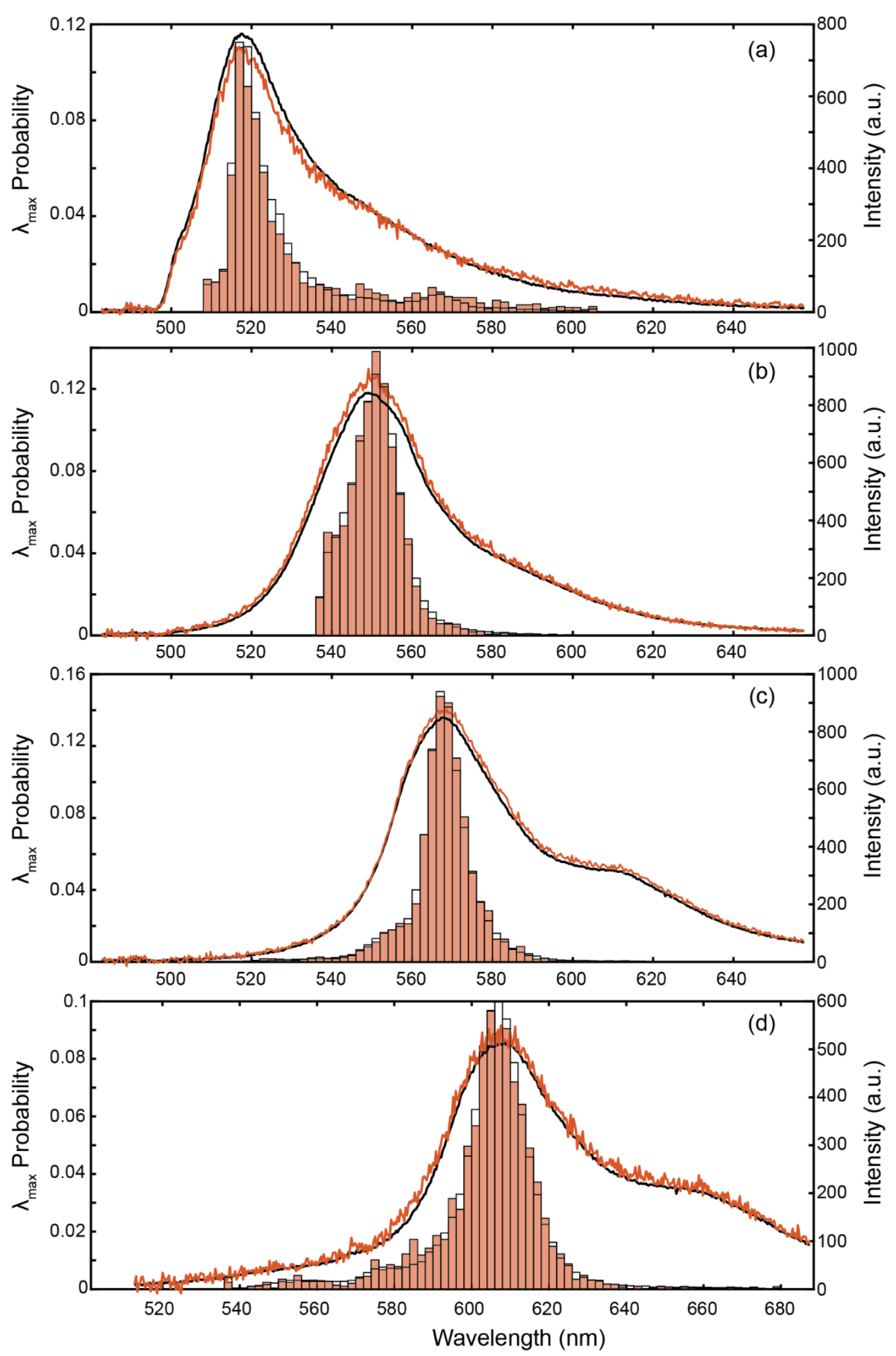

Figure S3. Emission maximum distributions (left axis, histograms) and average emission spectra (right axis, curves) for all molecules with $r>700 \mathrm{~nm}$ (black curve, white histograms) and $r<400 \mathrm{~nm}$ (orange curves, orange histograms) for each dye: (a) BDP-FL, (b) BDP-R6G, (c) Cy3, and (d) Cy3.5. Here, $r$ is the dye/AuNP separation distance (main text, Fig. 1b). 

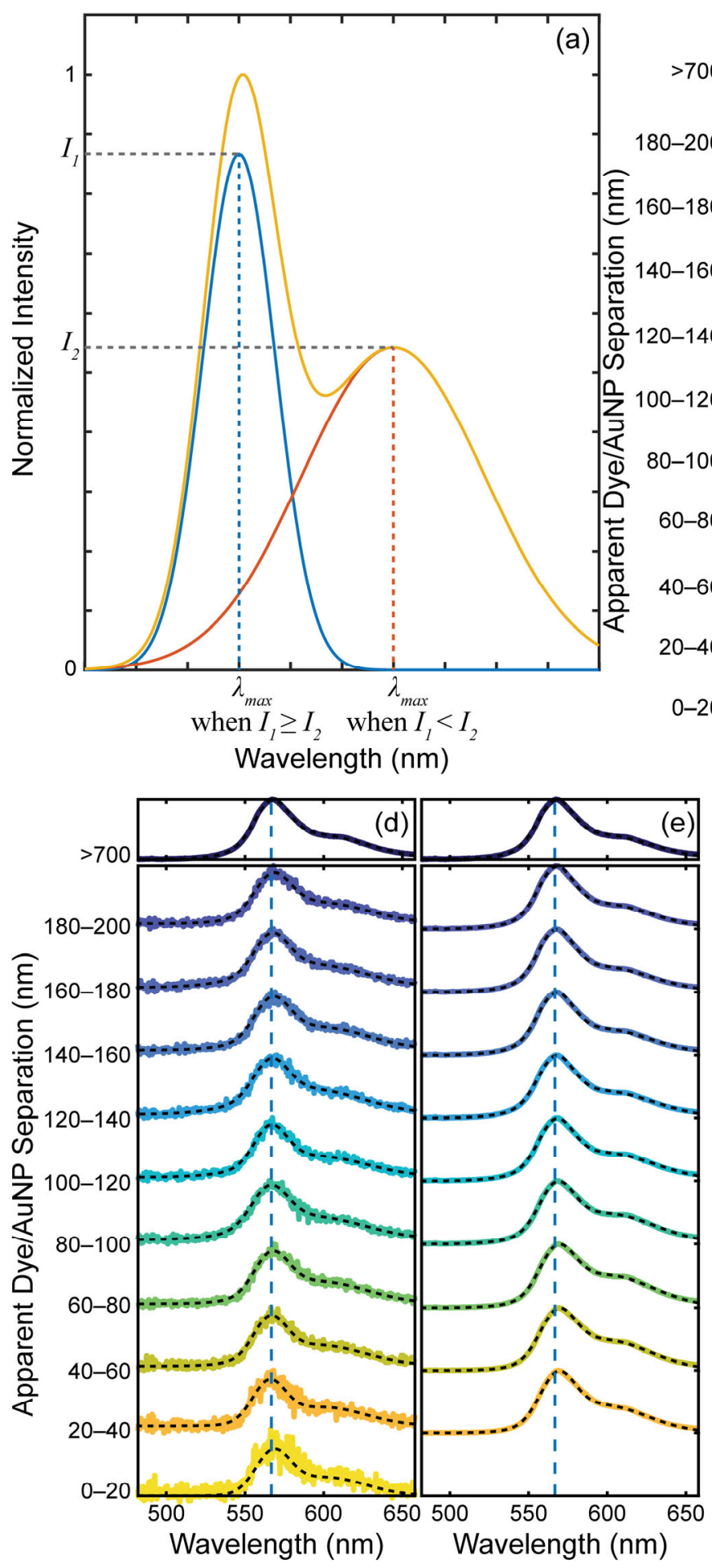
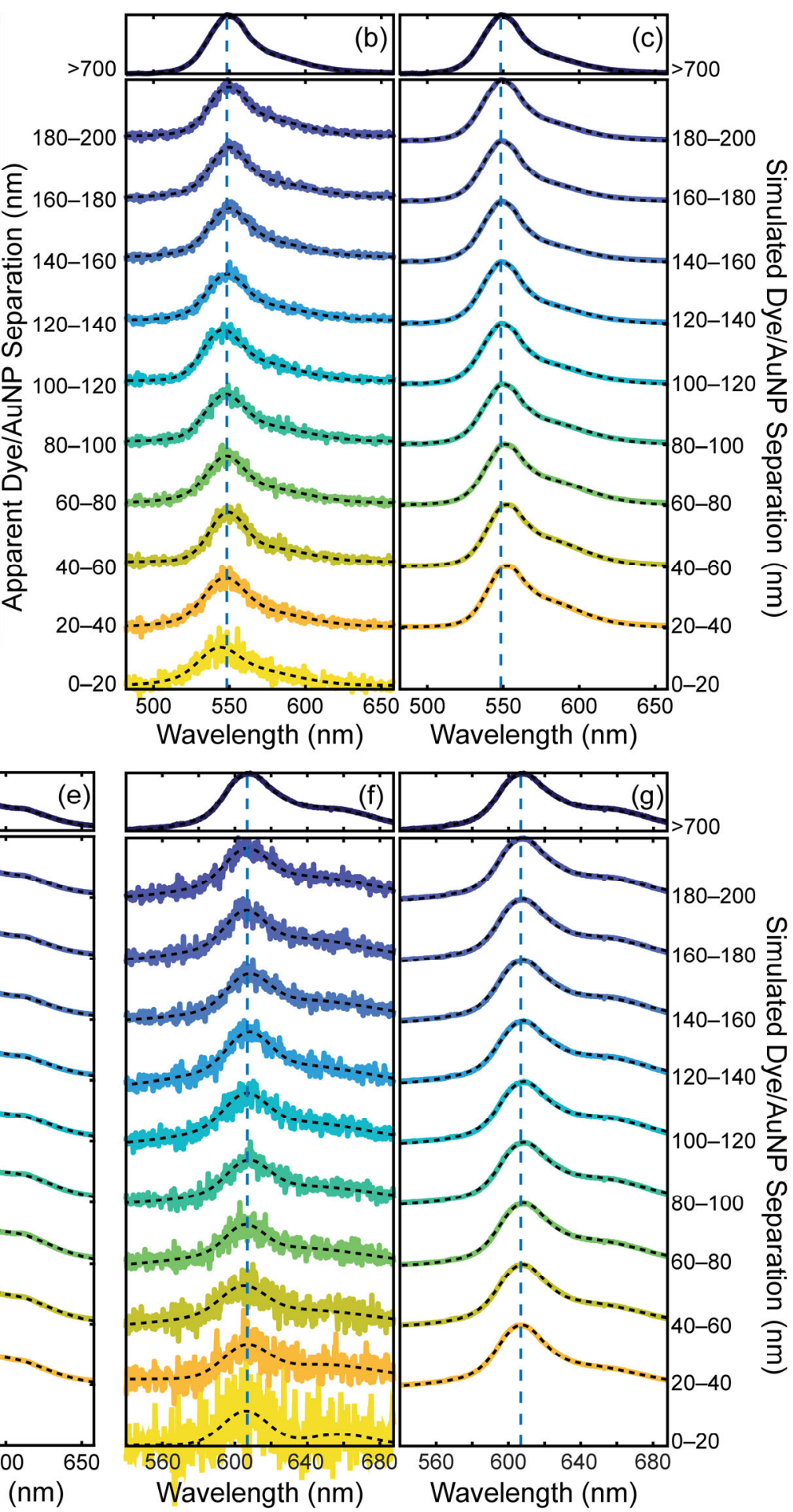

Figure S4. (a) Schematic of the two-term Gaussian (Eq. (1)) fit to a single-molecule spectrum. The full fit (yellow curve) is the sum of a Gaussian function with intensity $I_{1}$ (blue curve) and a Gaussian function with intensity $I_{2}$ (red curve). The maximum wavelength, $\lambda_{\max }$, is the peak of the blue curve when $I_{1} \geq I_{2}$ (blue dashed line), and the peak of the red curve is $\lambda_{\max }$ when $I_{1}<I_{2}$ (red dashed line).

$(b-g)$ Average emission spectra for dyes at different dye/AuNP distances for data (b, d, and $f$ ) and simulation (c, e, and g). Vertical dashed lines show the intrinsic emission maximum of each dye. $b$ and $c$ are BDP-R6G, $\mathrm{d}$ and e are $\mathrm{Cy} 3$, and $\mathrm{f}$ and $\mathrm{g}$ are $\mathrm{Cy} 3.5$. 


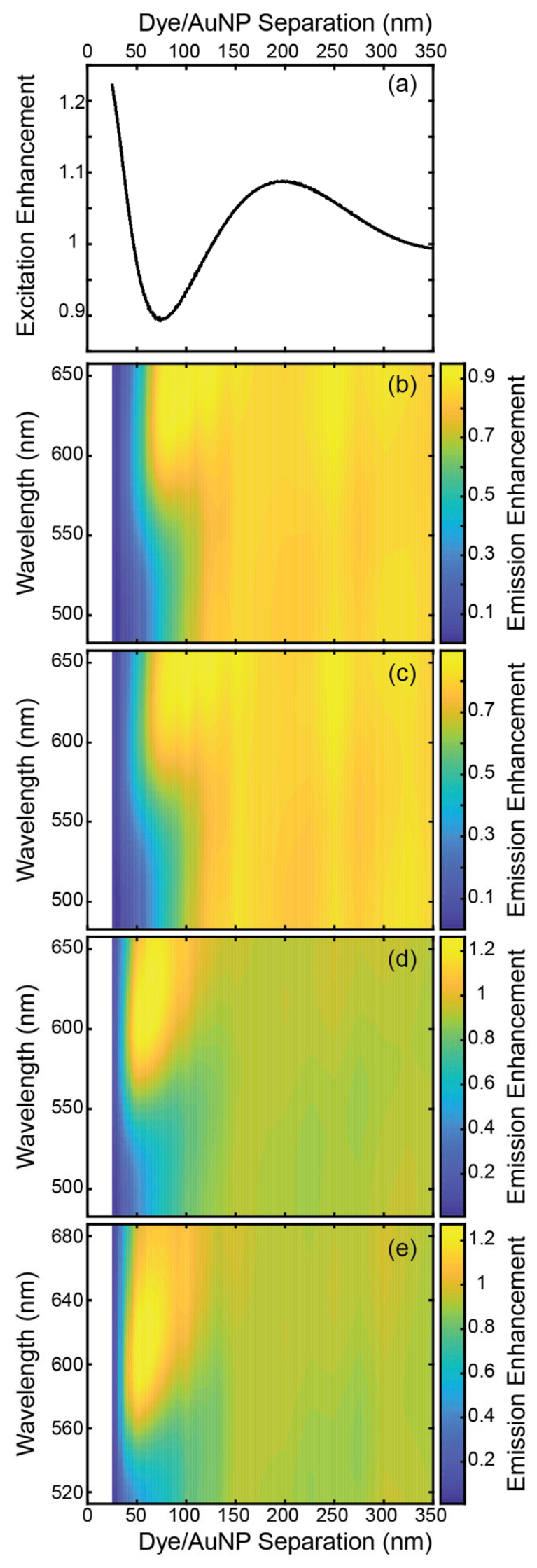

Figure S5. (a) Excitation enhancement function, $g_{\text {ex }}$ in Eq. (S2), vs. dye/AuNP separation at $488 \mathrm{~nm}$ for a $95-$ $\mathrm{nm}$ diameter gold sphere.

$(\mathrm{b}-\mathrm{e})$ Emission enhancement function, $g_{\text {em }}$ in Eq. (S3), for (b) BDP-FL, (c) BDP-R6G, (d) Cy3, and (e) Cy3.5. The enhancement factors remain relatively flat in the wavelength dimension for dye/AuNP separations, $r>$ $150 \mathrm{~nm}$, but have a strong wavelength dependence for separations, $r<150 \mathrm{~nm}$. 


\section{Supporting Movie Caption}

Video S1. Hyperspectral microscopy of single BDP-FL dye molecules adsorbing on a microscope coverslip near an AuNP (corresponding to Fig. 2a-d). Left: imaging channel shows AuNP photoluminescence and BDP-FL fluorescence images. The imaging channel is $9.5 \mu \mathrm{m} \times 1 \mu \mathrm{m}$. Right: spectral channel shows the corresponding photoluminescence and fluorescence spectra. The spectral channel $x$ axis is in wavelength space (range: $483-658 \mathrm{~nm}$ ) and its $y$ axis is in position space (height: 9.5 $\mu \mathrm{m})$. The movie is playing in real time at $3.33 \mathrm{fps}$.

\section{Supporting References}

(1) Ringler, M.; Schwemer, A.; Wunderlich, M.; Nichtl, A.; Kuerzinger, K.; Klar, T. A.; Feldmann, J. Shaping Emission Spectra of Fluorescent Molecules with Single Plasmonic Nanoresonators. Phys. Rev. Lett. 2008, 100, 203002.

(2) Wertz, E.; Isaacoff, B. P.; Flynn, J. D.; Biteen, J. S. Single-Molecule Super-Resolution Microscopy Reveals How Light Couples to a Plasmonic Nanoantenna on the Nanometer Scale. Nano Lett. 2015, 15, 2662-2670.

(3) Wertz, E. A.; Isaacoff, B. P.; Biteen, J. S. Wavelength-Dependent Super-Resolution Images of Dye Molecules Coupled to Plasmonic Nanotriangles. ACS Photonics 2016, 3, 1733-1740. 